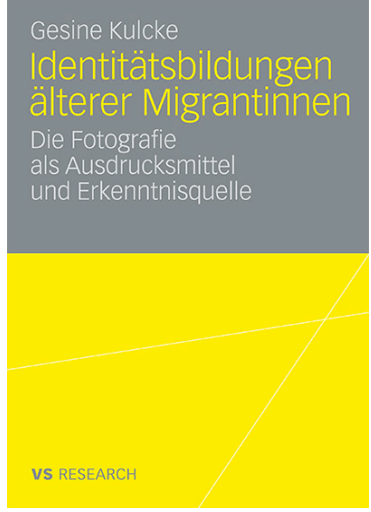

\section{Migrantinnen machen Fotos und entdecken dabei sich selbst}

Man muss die immer wieder aufkommenden Debatten um Deutschland als Einwanderungsland nicht einmal bemühen, um die Relevanz der vorliegenden Untersuchung zu belegen. Und bevor man den Fehler begeht, eine durch Vorurteil und Stereotypie vorbestimmte Außensicht auf die Migranten vorzunehmen, beginnt die Arbeit von Gesine Kulcke mit der Frage nach der Identität von älteren Migrantinnen. Sie leitet die notwendigen Konzepte der Identität im Wesentlichen von dem Soziologen Pierre Bourdieu, dem Psychologen Erik Erikson oder dem Philosophen Jürgen Habermas her. Die Autorin interessiert sich insbesondere für die Wahrnehmung von älteren Migrantinnen, betrachtet die Erfahrung der Migration als tiefgreifenden biografischen Bruch und diagnostiziert dabei eine Identitätskrise. Die Arbeit sieht ihre Zielsetzung in der pädagogischen Intervention mit älteren Migrantinnen, die in besonderem Maße ein Schicksal als orientierungslose und handlungsunfähige Menschen mit ökonomischen, sprachlichen und sozialen Defiziten führen. Migrationshintergrund, Weiblichkeit und Alter sind für sich genommen jeweils Merkmale, die das Interesse der Pädagogik rechtfertigt, und bei der hier ausgewählten Gruppe kulminieren.

Identität wendet den Blick auf die Innensicht des Individuums. Doch hier verstellt zunächst ein methodisches Problem die weitere Erkenntnis: Wie ist ein Selbstbild zu ermitteln? Das Selbstbild - das ist eine Voraussetzung der Arbeit - hat Konsequenzen auf die Art, wie die soziale Umwelt jeweils indivi- 


\section{Rezensionen}

duell wahrgenommen wird. Der Begriff der Weltanschauung kann hier wörtlich genommen werden. Dies hat eine methodische Frage zur Folge: Wie kann man den Blick auf die Welt erkennen und dabei identifizieren, was und wie das Individuum wahrnimmt? Was erregt seine Aufmerksamkeit? Wie ordnet es seine Welt? Was hat Bedeutung? Hier bietet Gesine Kulcke eine kreative Lösung: Sie lässt ältere Migrantinnen die Orte fotografieren, welche für sie von persönlicher Bedeutung sind. Nun kann man als Forscher diese Fotografien zunächst mit den Mitteln der Bildanalyse betrachten und er kann anschließend darüber mit der Fotografin ins Gespräch kommen. Fotografien repräsentieren den subjektiven Blick. Sie stellen dar, was die Fotografierenden auswählen. Diese Auswahl ist selten rational bestimmt, sondern folgt einer spontanen Eingebung. Die Auswahl des Bildausschnittes ist nicht zufällig, denn die Fotografien beziehen Kontexte mit ein oder lassen sie aus, wählen den Standpunkt, setzen Objekte ins Zentrum oder an den Rand. Sie repräsentieren emotional aufgeladene Objekte und lassen sich damit als Akt der Selbstoffenbarung verstehen. Gesine Kulcke bat eine Reihe von Migrantinnen, die sie in sozialen Projekten und Sprachkursen rekrutierte, einen bedeutsamen Ort in der zweiten Heimat zu fotografisch festzuhalten. Die Umsetzung dieser Aufgabe ist zwar ein bewusster und gelenkter Vorgang, dennoch ist die Entscheidung dem Bewusstsein nicht unbedingt zugänglich und darin liegt der Charme der Methode. Der erste Schritt der Analyse besteht in der Bildanalyse mit den für diesen Gegenstand zur Verfügung stehenden Instrumenten wie Perspektive, Mittelpunkt, Bildlinien, Formen und Farben. Der zweite Schritt, der sich im Gespräch über die Fotografien darstellt, ist unerlässlich, um die Motivationen und Hintergrundgeschichten der jeweiligen Auswahl zu erfahren, die aus der Bildbetrachtung selbst nicht zu erschließen sind. 
Interessant ist, wie sich zwei Zugänge - Bildbetrachtung und Interview - auf kluge Weise verbinden. Der Vorgang des Fotografierens schaltet vorgeschaltete Rationalisierungen weitgehend aus und das Interview, das sich vordergründig auf die Fotografie fokussiert, ermittelt die verdeckten Bedeutungen. Die Arbeit zieht ihren Wert nicht nur aus der Auswahl des Gegenstandes, sondern aus der methodischen Herangehensweise. Sie führt ein Verfahren vor, das ohne weiteres auch auf andere Gegenstandbereiche der Identitätsbildung anzuwenden ist.

Clemens Schwender 